\title{
A new data acquisition and processing system for UAVSAR
}

\author{
Chot Hun Lima), Chee Siong Lim ${ }^{\mathrm{b})}$, Ming Yam Chua, \\ Yee Kit Chan, Tien Sze Limc), and Voon Chet Koo \\ Faculty of Engineering \& Technology, Multimedia University \\ Jalan Ayer Keroh Lama, Melaka 75450, Malaysia \\ a)chlim@mmu.edu.my \\ b)cslim@mmu.edu.my \\ c) tslim@mmu.edu.my
}

\begin{abstract}
Synthetic Aperture Radar (SAR) is widely known as a high resolution imaging system in microwave remote sensing. Large number of frequency-modulated received echoes must be acquired in real time. A typical SAR data acquisition unit (DAQ) involves high speed analog-to-digital conversion, front-end pre-processing, and data recording. Subsequent processes consist of computationally intensive digital signal processing for image formation. In this paper, an efficient data acquisition and SAR processing method is proposed. It is based on a modified discrete Fourier Transform algorithm, which requires lesser system's computational load as compared to conventional Fast Fourier Transform. The proposed system has been implemented on an UAVSAR (Unmanned Aerial Vehicle SAR) and the flight tests have shown promising results for real-time imaging.
\end{abstract}

Keywords: UAVSAR, modified discrete Fourier Transform, DAQ Classification: Microwave and millimeter wave devices, circuits, and systems

\section{References}

[1] M. Soumekh, Synthetic Aperture Radar Signal Processing with MATLAB Algorithm, John Wiley \& Sons, Inc, New York, 1999.

[2] I. G. Cumming, F. H. Wong, Digital Processing of Synthetic Aperture Radar Data: Algorithms and Implementation, Artech House, 2005.

[3] P. A. Rosen, S. Hensley, K. Wheeler, G. Sadowy, T. Miller, S. Shaffer, R. Muellerschoen, C. Jones, S. Madsen, and H. Zebker, "UAVSAR: New NASA Airborne SAR System for Research," IEEE Aerosp. Electron. Syst. Mag., vol. 22, no. 11, pp. 21-28, Dec. 2007.

[4] C. Simon-Klar, L. Friebe, H. Kloos, H. Lieske, W. Hinrichs, and P. Pirsch, "A Multi DSP Board for Real Time SAR Processing using the HiPARDSP 16," Proc. IEEE Geoscience and Remote Sensing Symposium, 2002, IGARSS'02, vol. 5, pp. 2750-2752, 2002.

[5] C. Le and S. Chan, "Onboard FPGA-based SAR processing for future spaceborne systems," Proc. IEEE Radar Conference, pp. 15-20, 2004.

[6] Y. K. Chan, V. C. Koo, B. K. Chung, and H. T. Chuah, "Modified Algo- 
rithm for Real Time SAR Signal Processing," Proceeding of Progress In Electromagnetics Research C, vol. 1, pp. 159-168, 2008.

\section{Introduction}

Synthetic Aperture Radar (SAR) has been an important tool for civilians and military applications for many years. Its wide ground surface coverage with high resolution and all weather capabilities make it one of the most popular imaging systems for remote sensing. Conventional SARs are typically mounted on aircraft or spacescraft $[1,2]$. Lately, there is an increase popularity using Unmanned Aerial Vehicles (UAVs) for SAR missions [3], mainly because there is no risk of losing human life and it offers a cost effective solution for timely operation. Due to limited payload of UAV (which is normally lesser than $20 \mathrm{~kg}$ ), an onboard UAVSAR system must be compact in size and at the same time possess fast processing capabilities for real time implementation. These lead to the motivation of designing new SAR data acquisition system (DAQ) and processing system which is fast in computational speed, compact in size with reasonable cost.

Conventional designs use high speed dedicated chipsets, such as the digital signal processor (DSP) [4] and the field-programmable gate array (FPGA) [5] for UAVSAR system. However, there exist certain drawbacks in these designs, such as no real time image output and long processing time. By using these conventional designs, the formulation of SAR image can only be acquired through post-processing using a computer (PC).

This work focuses on designing a new efficient SAR data acquisition and processing system that aims to provide a better UAVSAR data processing efficiency. The new system is also compact in size, and it tackles the above mentioned conventional designs' drawbacks for UAVSAR system. The processing efficiency is enhanced by two approaches. The first approach is achieved by implementing a modified Discrete-Fourier Transform (DFT) algorithm [6] to performed UAVSAR data processing, while the second approach is done through the enhancement of the DAQ's hardware design. With these approaches the designed system is able to record and process the SAR raw data concurrently, and it provides processed image output in real time.

The rest of the paper is organized as follow. Section 2 provides an in-depth discussion on the modified DFT algorithm, with comparison to conventional DFT algorithm. Section 3 outlines the DAQ design and its processing efficiency's enhancement. Section 4 discusses the results by implementing the modified DFT algorithm and the DAQ system for UAVSAR data processing. Finally, section 5 concludes the paper.

\section{Modified DFT algorithm for UAVSAR processing}

UAVSAR signal processing is commonly separated into range compression and azimuth compression, as shown in Fig. 1. Both compressions are per- 


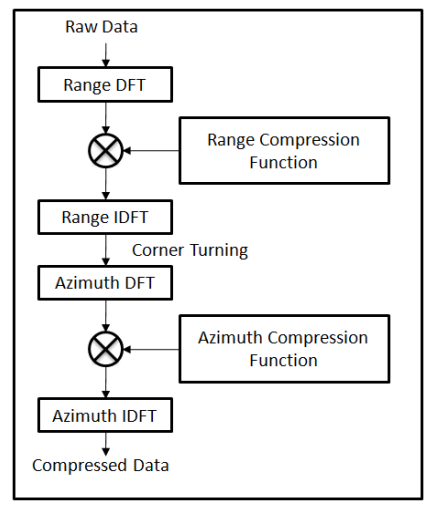

Fig. 1. UAVSAR Image Formation Block Diagram

formed in frequency domain through DFT (Discrete Fourier Transform) and IDFT (Inverse-Discrete Fourier Transform) to ease the computational load. Since the efficiency of UAVSAR processing relies heavily on DFT and IDFT computation, an optimized DFT and IDFT algorithm will definitely improved the SAR processing performance.

This section compares a conventional UAVSAR DFT processing algorithm with the modified-DFT algorithm. Based on the analysis the modifiedDFT is much more suitable for real time implementation as compared to conventional algorithm.

\subsection{Conventional DFT}

The conventional discrete Fourier-Transform (DFT) pair of a discrete-time signal $s(n)$ with length $N$ can be expressed as:

$$
\begin{aligned}
& s(n)=\frac{1}{N} \sum_{k=0}^{N-1} S(k) \exp \left\{j \frac{2 \pi k n}{N}\right\}, k=0,1, \ldots \ldots, N-1 \\
& S(k)=\sum_{n=0}^{N-1} s(n) \exp \left\{-j \frac{2 \pi k n}{N}\right\}, n=0,1, \ldots \ldots, N-1
\end{aligned}
$$

It is possible to expand (1) into two dimensions with $N_{1}$ and $N_{2}$ as lengths of each dimension:

$$
\begin{aligned}
s\left(n_{1}, n_{2}\right) & =\frac{1}{N_{1} N_{2}} \sum_{k_{1}=0}^{N_{1}-1} \sum_{k_{2}=0}^{N_{2}-1} S\left(k_{1}, k_{2}\right) \exp \left\{j 2 \pi\left(\frac{k_{1} n_{1}}{N_{1}}+\frac{k_{2} n_{2}}{N_{2}}\right)\right\}, \\
k_{1}, k_{2} & =0,1, \ldots \ldots, N-1 \\
S\left(k_{1}, k_{2}\right) & =\sum_{n_{1}=0}^{N_{1}-1} \sum_{n_{2}=0}^{N_{2}-1} s\left(n_{1}, n_{2}\right) \exp \left\{-j 2 \pi\left(\frac{k_{1} n_{1}}{N_{1}}+\frac{k_{2} n_{2}}{N_{2}}\right)\right\}, \\
k_{1}, k_{2} & =0,1, \ldots \ldots, N-1
\end{aligned}
$$

From (2), the total computational load of an $N$-point DFT is equal to $N^{2}$ complex multiplications and $N(N-1)$ complex additions, with one complex multiplication consists of four real multiplications and two real additions, and one complex addition equal to two real additions. Therefore, each $N$ point DFT consists of $4 N^{2}$ numbers of real multiplications and $4 N^{2}-2 N$ of 
real additions. When $N$ is of power of 2, there exists a method known as the Fast-Fourier Transform (FFT) which reduces the computational load to $N \log _{2}(N)$. Any non radix-2 matrices can be zero padded to radix-2 matrix for FFT and IFFT. Equation (4) shows the computational load of $N$-point FFT.

$$
\begin{aligned}
& F F T_{M}=2 N \log _{2} N \\
& F F T_{A}=3 N \log _{2} N
\end{aligned}
$$

\subsection{Modified DFT algorithm}

Consider a signal $g(n)$ with data length of $N$, where $N \neq 2^{M}$. Let $N=N_{1} N_{2}$ so that $g(n)$ is separated into a $N_{1}$ arrays with $N_{2}$ data length [6], as given:

$$
g(n)=\left[\begin{array}{ccccc}
x(0) & x(1) & x(2) & \ldots & x\left(N_{2}-1\right) \\
x\left(N_{2}\right) & x\left(N_{2}+1\right) & x\left(N_{2}+2\right) & \ldots & x\left(2 N_{2}-1\right) \\
x\left(2 N_{2}\right) & x\left(2 N_{2}+1\right) & x\left(2 N_{2}+2\right) & \ldots & x\left(3 N_{2}-1\right) \\
\vdots & \vdots & \vdots & \vdots & \vdots \\
x\left(\left(N_{1}-1\right) N_{2}\right) & x\left(\left(N_{1}-1\right) N_{2}+1\right) & x\left(\left(N_{1}-1\right) N_{2}+2\right) & \ldots & \left.x\left(N_{1} N_{2}-1\right)\right]
\end{array}\right.
$$

where $n=N_{1} n_{2}+n_{1}$ for $0 \leq n_{1} \leq N_{2}-1$ and $0 \leq n_{2} \leq N_{1}-1$.

An $N$-point two-dimensional DFT can thus be expressed as follows:

$$
\begin{aligned}
G(k) & =\sum_{n_{1}=0}^{N_{1}-1} \sum_{n_{2}=0}^{N_{2}-1} g(n) e^{-2 \pi \frac{k n}{N}} \\
& =\sum_{n_{1}=0}^{N_{1}-1} \sum_{n_{2}=0}^{N_{2}-1} g\left(N_{1} n_{2}+n_{1}\right) e^{-2 \pi \frac{\left(k_{2}+N_{2} k_{1}\right)\left(N_{1} n_{2}+n_{1}\right)}{N_{1} N_{2}}} \\
& =\sum_{n_{1}=0}^{N_{1}-1}\left\{\sum_{n_{2}=0}^{N_{2}-1}\left[g\left(N_{1} n_{2}+n_{1}\right) e^{-2 \pi \frac{n_{2} k_{2}}{N_{2}}}\right] e^{-2 \pi \frac{k_{2} n_{1}}{N_{1} N_{2}}}\right\} e^{-2 \pi \frac{k_{1} n_{1}}{N_{1}}}
\end{aligned}
$$

where $k=N_{2} k_{1}+k_{2}$ for $0 \leq k_{1} \leq N_{1}-1$ and $0 \leq k_{2} \leq N_{2}-1$.

Therefore, by using the modified DFT algorithm, it is possible to process the DFT of a signal with data length $N$ in three steps: $N_{1}$ number of $N_{2^{-}}$ point DFT, followed by $N_{2}$ numbers of $N_{1}$ point complex multiplications, and finally $N_{2}$ numbers of $N_{1}$-point DFT. Equation (6) shows the computational load of $N$-point modified DFT algorithm.

$$
\begin{aligned}
& \operatorname{MDFT}_{M}=2 N_{1} N_{2} \log _{2} N_{2}+4 N_{2} N_{1}^{2}-4 N_{1} N_{2}+4 N_{2} \\
& \operatorname{MDFT}_{A}=3 N_{1} N_{2} \log _{2} N_{2}+4 N_{2} N_{1}^{2}-4 N_{1} N_{2}+2 N_{2}
\end{aligned}
$$

In summary, it is possible to increase the computational efficiency by choosing $N_{2}$ to be a radix-2 number for DFT utilization, and $N_{2}$ should always be much larger than $N_{1}$.

\section{Data acquisition unit (DAQ) design for UAVSAR}

Implementation of UAVSAR DAQ and processing system is always a challenging task, lies on the fact that a huge amount of data is to be simultaneously recorded and processed within a few microseconds. Conventional real 


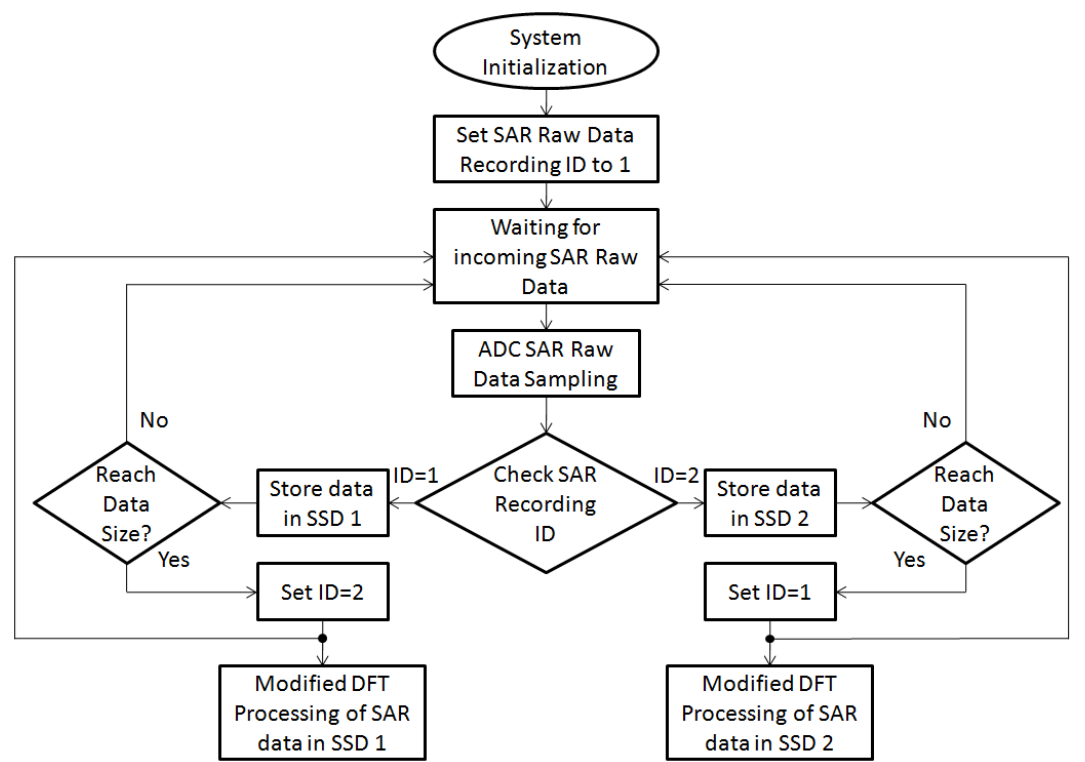

Fig. 2. Block Diagram of Enhanced UAVSAR Data Recording and Processing System

time SAR data processing is commonly implemented by properly sub-divide the SAR processing task into multiple sessions and realized through multiple DSPs [4] or FPGAs [5].

In this paper, a real time UAVSAR recording and processing system were successfully implemented using only a single HPC as the recording and processing tool. Fig. 2 shows the block diagram of the enhanced UAVSAR data acquisition and processing system. It is worth mentioning that UAVSAR raw data is acquired using a high speed 12-bits dual-channel analog-to-digital converter $(\mathrm{ADC})$ with a designed data rate of $7.5 \mathrm{Mbytes} / \mathrm{s}$. After the conversion, the ADC stored the data in HPC's Random Access Memory (RAM) temporarily, where the HPC will excerpt the information out of the RAM and saved it into local mass storage device. Here lies the first bottleneck of the designed system, where a typical magnetic hard disk should not be used in UAV flight test due to the vibration of the flight might potentially damaged the hard disk's spinning disk with its read/write heads. This bottleneck can be resolve by replacing the magnetic hard disk with solid-state drive (SSD). A SSD is a mass storage device uses microchip to store data. It contains no moving part during data retaining as compared to magnetic hard disk's spinning disk, and its data rate is also faster than magnetic hard disk. The second bottleneck lies on the physical data bus congestion in real time UAVSAR recording and processing application. However, enhancement can be made to resolve this bottleneck by proper arrangement of two SSDs into the system. As shown in Fig. 2, the HPC is designed in such a way that the UAVSAR raw data recording and processing always taking place at different SSD, thus reducing the SSD's data bus congestion and enhancing the UAVSAR data processing efficiency. 


\section{System implementation and result analysis}

This section outlines the implementation of the modified DFT algorithm to the UAVSAR system. UAVSAR flight experiments had been conducted in December 2010 at Mersing, Johor, Malaysia. These experiments implemented both the modified DFT algorithm and the DAQ design. Fig. 3 depicts the overall system implementation block diagram for SAR processing, including the screenshot of the DAQ's Graphical User Interface (GUI). During the Mersing flight, 6 flight measurements were conducted and a total volume of 88 GB SAR data were collected. Fig. 3 also shows one of the processed SAR images with comparison to the Google Earth map of the same site.

During the flight mission, the UAV had a nominal flight altitude of $1000 \mathrm{~m}$, and the UAV's chirp generator had a transmission rate of $640 \mathrm{~Hz}$. The flight altitude of $1000 \mathrm{~m}$ requires the ADC to acquire 3072 range points every $1 / 640$ second. The azimuth points is chosen to be 1400 to benefit from the enhanced features discussed in Fig. 2. Thus the system is able to process the UAVSAR raw data once 3072 range points and 1400 azimuth points were collected. The system will swap the recording SSD during the UAVSAR data processing, and vice versa.

As mentioned previously, UAVSAR image formation requires huge computational power on DFT and IDFT. Any mean of reducing the computational loads (which in turn improve processing speed) while preserving its output image quality indicate an improvement in the system. The processed image shown in Fig. 3 was the SAR processing result of either using conventional algorithm or modified DFT algorithm. Conventional algorithm using

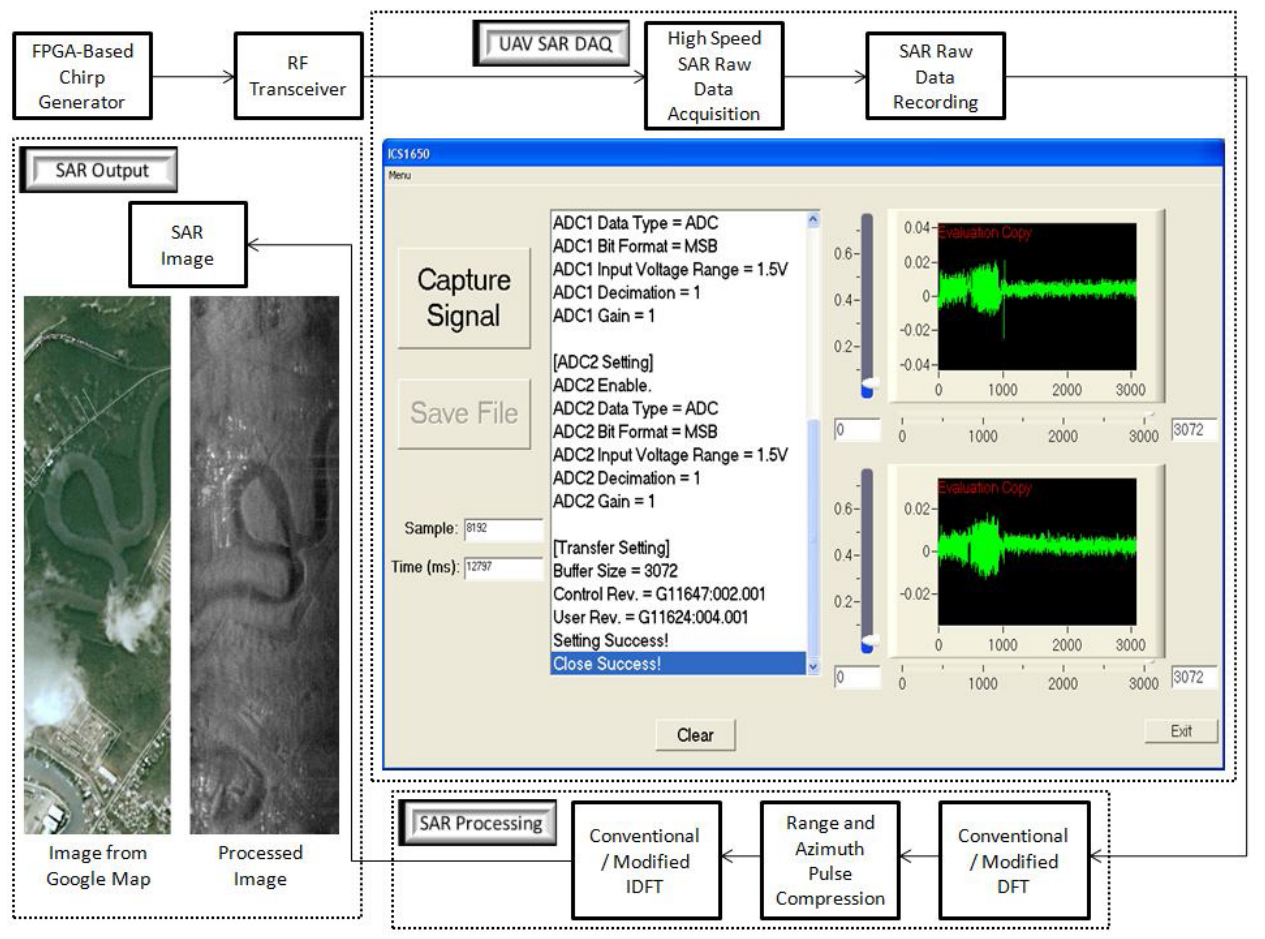

Fig. 3. DAQ and Modified DFT implementation for real time SAR Processing (with output image) 
Table I. Computational Operations Comparison based on 1400 X 3072 Array of Data

\begin{tabular}{|l|l|c|c|}
\hline & & Real Multiplication & Real Addition \\
\hline \multirow{3}{*}{$\begin{array}{l}\text { 1400 Data Points } \\
\text { Azimuth Direction }\end{array}$} & $\begin{array}{l}\text { Conventional FFT } \\
\text { Algorithm }\end{array}$ & 45,056 & 67,584 \\
\cline { 2 - 4 } & $\begin{array}{l}\text { Modified DFT } \\
\text { Algorithm }\end{array}$ & 41,984 & 54,784 \\
\cline { 2 - 4 } & \% of Improvement & $6.81 \%$ & $18.9 \%$ \\
\hline \multirow{3}{*}{$\begin{array}{l}\text { (Range Direction) } \\
\text { Range }\end{array}$} & $\begin{array}{l}\text { Conventional FFT } \\
\text { Algorithm }\end{array}$ & 98,304 & 147,456 \\
\cline { 2 - 4 } & $\begin{array}{l}\text { Modified DFT } \\
\text { Algorithm }\end{array}$ & 90,112 & 118,784 \\
\cline { 2 - 4 } & $\%$ of Improvement & $8.00 \%$ & $19.4 \%$ \\
\hline \multirow{2}{*}{$\begin{array}{l}\text { Total Number of } \\
\text { operation for 1400 }\end{array}$} & FFT \& IFFT & $552,075,264$ & $828,112,896$ \\
\cline { 2 - 4 } X 3072 Data Points & Modified DFT \& IDFT & $510,263,296$ & $669,188,096$ \\
\cline { 2 - 4 } & $\%$ of Improvement & $7.57 \%$ & $19.19 \%$ \\
\hline
\end{tabular}

DFT and IDFT for UAVSAR image formation requires zero padding for both range and azimuth points to its closest radix of 2 , which are equivalent to 4096 range points and 2048 azimuth points. A total number of 1024 range points and 648 azimuth points are zero padded for range DFT/IDFT and azimuth DFT/IDFT. As for the modified DFT/IDFT algorithm, let the range points be decomposed as $N=1024 \times 3=3072$ and azimuth points be decomposed as $N=512 \times 3=1536$. Hence only 136 zeros were padded into azimuth points and no zero padding was required for range points.

Table I presents the comparison of computational loads between the conventional algorithm and the modified algorithm. Equation (3) and (6) were used for the load computation. The results indicate significant improvement in computational efficiency of modified DFT algorithm over the conventional DFT algorithm, thus it achieve faster processing speed as compared with conventional system.

\section{Conclusion}

This paper presents a new approach for improved UAVSAR data acquisition and processing using a modified DFT algorithm together with an enhanced DAQ system. It is a compact and low cost solution by using commonly available hardware platform and software tools. Analysis and result show the applicability and efficiency of the system to be implemented on a real time UAVSAR system.

\section{Acknowledgments}

This work is partially funded by the Malaysian Remote Sensing Agency (ARSM). 